A. Duncan, MBChB, FCAP, Director, Special Haemostasis Labolatory, Emory University Hospital, Atlanta Georgia, GA 30322, USA

\title{
THE CASE FOR VENOUS THROMBOEMBOLISM PROPHYLAXIS IN AFRICA
}

\author{
A. DUNCAN
}

\section{SUMMARY}

\begin{abstract}
While venous thromboembolism (VTE) is a well recognised occurrence in clinical practice in the developed world, with event rates of at least 2-3 million per year, little attention is paid to this entity in the developing world where the burden of infectious diseases and limited access to care have not recognised VTE as a significant cause of morbidity and mortality. The opportunity for Africa to do better as the inevitable recognition of the consequence of VTE becomes more apparent is available using basic tools and therapies.
\end{abstract}

\section{INTRODUCTION}

Over the past 30 years, there has been an explosion in the recognition and quantization of VTE cases in the developed world. In the US alone, there are reported to be 2-3 million deep venous thrombosis (DVT) cases and 600,000 cases of pulmonary embolism (PE) per year, with one third of the latter being fatal. Within the past 10 years data has evolved to show that $70 \%$ of VTEs in hospital occur in medical patients and that $10 \%$ of hospital deaths are due to PE (1). This was surprising since most of the studies on the advantages of VTE prophylaxis done in the 1970s and $80 \mathrm{~s}$, focused on the orthopaedic population, especially joint replacement, where it was known that patients had very high rates of complicating DVT, if not given anticoagulant prophylaxis. The use of VTE prophylaxis in a wide spectrum of surgical patients became the norm in Europe and North America. In this respect, low molecular weight heparins, (LMWH) and the synthetic indirect Factor Xa inhibitor, fondaparinux have proved effective as prophylactic agents for VTE in surgical patients. Prophylaxis led to significant reduction in morbidity and mortality from VTE. However little attention was paid to the medical patient population until the start of this millennium.

\section{VTE IN MEDICAL PATIENTS}

The impact of the clinical and economic burden on US Healthcare of VTE in medical patients has led to some of the major regulatory authorities, namely The Joint Commission (TJC) and the National Quality Forum, (NQF) to endorse standardised VTE protocols for prophylaxis. These were introduced in 2008 because these bodies TJC/NQF identified that VTE, was the number one cause of preventable morbidity and mortality in US healthcare $(2,3)$. There was also an anticipated economic gain predicted if these measures were implemented. While the decrease in VTE rates from prophylaxis in the surgical patient population was known to be high, many clinicians assumed that the potential risk of bleeding would be higher in the medical patients population because of multiple co-morbidities. They feared the risk of bleeding in these medical patients would mitigate or simply prevent the use of an anticoagulant, even in prophylactic doses.

Risk factors associated with VTE include immobilisation, infection, heartfailure, and respiratory problems, active or underlying malignancy, increased age, priorVTE and underlying congenital or acquired thrombophilia. An important adjunct is that these factors are synergistic and the more risk factors present, the higher the VTE risk (4). Ironically, despite the disease burden of HIV infection, these patients do not have a signifiantly higher rate of VTE than non infectious medical patients. While many of the aforementioned risk factors are less relevant in Africa, as healthcare progresses and hopefully the disease burden imposed by infections such as malaria and HIV decreases, the impact of common malignancies such as breast cancer will increase. Concomitant with that increase and increased life expectancy will be an inevitable increased awareness of VTE (5). One area with a known increased risk is the pregnant woman especially in the past partum period where PE is the most likely cause of obstetric sudden death in the developed world. In much of Africa, where obstetrical bleeding is a significant cause of mortality, VTE is not likely to be a focus of care. The use of anticoagulant prophylaxis requires input and oversight by physicians. Even in the US where 
the information and data to support the widespread use of VTE prophylaxis has existed for some time, only one third of patients in a recent study received prophylaxis at all, or were given prophylaxis of appropriate dose or adequate duration. In a very recent study, of 200,000 US medical patients, the overall VTE event rate was $5.6 \%$ with $80 \%$ having DVT only. Thirty six per cent of DVTs occurred in the first 30 days of hospitalisation, but a significant number occurred up to 60 days, confirming the need for appropriate duration of therapy. Cancer patients exhibited the highest risk with heart failure and lung problems also having high risks (5).

Another potential important health economic benefit is that in this medical patient group the recurrence rate for VTE is about $23 \%$ in a 50 month follow up. It is not difficult to see why the primary prevention of VTE also impacts on the significant cost of hospital readmission (4).

\section{VTE PROPHYLAXIS IN AFRICA}

How much of this information from the developed world has relevance for current clinical practice in Africa? This author is aware from his limited time in East Africa that VTE does exist and is recognised by most haematologists and specialist physicians, but rarely so by other generalist-physicians. Would there be significant and quantifiable patient benefit from more widespread use of VTE prophylaxis especially in the large national or regional hospitals? What are the challenges to implementation? In the developed world the availability oflow molecular weightheparin (LMWH) and fondaparinux drugs is ubiquitous and is cost effective for use widely. On the whole these anticoagulants require no laboratory monitoring and are therefore particularly suited to settings where rapid appropriate laboratory monitoring are not available. Given the huge burden of treating infectious diseases it is unlikely that providing prophylaxis widely with these anti coagulants could be justified. Traditional unfractionated heparin (UFH) is widely available and is inexpensive. However UFH requires monitoring with laboratory tests. A need therefore exists to consider use of LMWH and fondaparinux in settings where accurate laboratory monitoring is not available. UFH was used for prophylaxis for many years in the developed world before the widespread availability of LMWHand fondaparinuxUFHsteadily administered by pump is clinically effective and could provide a practical method to provide low cost prophylaxis in settings where reliable laboratory monitoring is available. Consideration of the role of LMWH and fondaparinux should be made, especially when pumps and laboratory monitoring facilities are unavailable. While skepticism may dictate that currently no cost justifiable case can be made for widespread VTE prophylaxis in Africa, this author believes that evolving medical practice in East Africa if only in the increasingly diagnosed cancer patients, would merit at least a pilot trial of heparin prophylaxis in a large medical centre. It seems to this author that East Africa could develop from the published world data and its own population of patients a simple cost effective strategy to minimise theinevitablehealthcare burden that VTE maybe imposing currently, and that it may impose in the future.

\section{REFERENCES}

1. Amin, A., Stemkowski, S., Lin, J., et al. Thromboprophylaxis rates in US medical centres: Success or failure. J. Thromb. Haemost. 2007; 5: 1610-1616.

2. Geerts, W.H., Pineo, G.F. and Heit, J.A. Prevention of VTE. Evidence based clinical practice guidelines. CHEST ( $8^{\text {th }}$ edition) 2008; 133 (16): 381S-452S.

3. McDougall, D.A., Feliu, A.L., Boccuzzi, S.J., et al, Economic burden of DVT and PE and post phlebitic syndrome. Am. J. Health. Syst. Pharm. 2006; 63 (20 suppl 6): S5-S15.

4. Alikan, R., Cohen, A.P., Combe, S., et al. MEDENOX study. Risk factors for VTE in hospitalised patients with acute medical illness. Arch. Inter. Med. 2004; 164: 963-968.

5. Spryopoulos, A.C., Hussein, M., Lin, J., et al. Rates of VTE occurrence in medical patients in the insured population. J. Thromb. Haemost. 2009; 102: 951-957. 